\title{
Free-space delay lines and resonances with ultraslow pulsed Bessel beams
}

\author{
Carlos J. Zapata-Rodríguez, Miguel A. Porras, and Juan J. Miret \\ Departamento de Óptica, Universidad de Valencia, Dr. Moliner 50, 46100 Burjassot, Spain \\ Departamento de Física Aplicada, Escuela Técnica Superior de Ingenieros de Minas, Universidad Politécnica de \\ Madrid, Rios Rosas 21, 28003 Madrid, Spain \\ Departamento de Óptica, Universidad de Alicante, P.O. Box 99, Alicante, Spain
}

\begin{abstract}
We investigate the ultraslow motion of polychromatic Bessel beams in unbounded, nondispersive media. Control over the group velocity is exercised by means of the angular dispersion of pulsed Bessel beams of invariant transverse spatial frequency, which spontaneously emerge from near-field generators. Temporal dynamics in transients and resonances over homogeneous delay lines (dielectric slabs) are also examined.

Society of America
\end{abstract}

\section{INTRODUCTION}

The speed of a light pulse propagating in an optical medium may vary significantly depending on the dispersion in the refractive index $n$ and ranges from subluminal to superluminal, and even negative, velocities. Ultraslow wave propagation refers to group velocities much smaller than $c$ or, equivalently, to group indices $n_{g}=n+\omega \partial_{\omega} n$ exceeding by far unity, which requires strong chromatic dispersion $\partial_{\omega} n$. Strong absorption under such conditions, however, brings severe limitations in the experimental observation of slow light, which has led to pursuit of quantum interference effects such as electromagnetically induced transparency or coherent population oscillations

Reduction of the group velocity was achieved much earlier in optical resonances. Structural dispersion in metallic hollow waveguides, or optical fibers, induces chromatic dispersion that allows for both subluminal and superluminal modes of propagation. Flatband regions of ultraslow propagation are found in the vicinity of bandgaps where, at the edges, modes with zero group velocity should exist. Slow-wave structure assemblies for use in traveling-wave tubes benefit from this phenomenon

Evidence of slow light phenomena in photonic crystals and their use in delay lines for optical buffering dispersion compensation and for enhanced light-matter interactions in nanophotonic circuits , have been described more recently.

In a rather different context undistorted wave transmission in free space with polychromatic Bessel beams has attracted considerable attention in recent years

Potential applications may be found in different research areas, e.g., remote sensing, high resolution imaging, impulse radar, plasma physics, directed energy transfer, and secure communications

In the absence of a medium or a structure it is the plane-wave angular dispersion that is at work in the control of the wave shape and its velocity Most of the research has been focused on superluminal diffraction-free solutions of the wave equation and on undistorted pulse beam propagation in dispersive dielectric media Although subluminal localized pulses have been reported previously , the characteristic features of pulsed Bessel beams (PBBs) with group velocity approaching zero have not been examined at length

We investigate in this paper slow-wave propagation of ultrashort PBBs in nondispersive bulk media, and free space as a particular case. Within the vast variety of nondiffracting PBBs presenting subluminal group velocity, we have focused on those with constant transverse spatial frequency , exhibiting therefore a high-pass semiinfinite band. Near the band edge we encounter proper conditions for ultraslow wave propagation. Far-field synthesis of PBBs usually relies on complex approaches such as dynamic aperturing . Alternatively, near-field generation of such wave packets is commonly performed by direct pulse-plane-wave diffraction with radial gratings or annular arrays . The characteristic periodic modulation of these diffractive optical elements allows the imprinting of a given spatial frequency, which is independent upon frequency, onto the wavefield. Also, the fundamental modes of surface-emitting semiconductor laser structures is of this kind Finally, excitation of stable $\mathrm{PBBs}$ driven by nonlinearities represents an attractive route

In this paper, pulse dynamics (in particular, transit times and pulse stretching) of PBBs traveling within nondispersive homogeneous material systems are thoroughly examined for applications such as all-optical transmission delay lines and resonant cavity confinement. We present analytical and numerical results of $\mathrm{PBBs}$ propagating at group indices ranging from 8 in glass microlines of $20 \mu \mathrm{m}$ for few-cycle wave packets, up to $n_{g}=1000$ for resonant 
optical $20 \mathrm{~ns}$ long pulses in a plane-parallel cavity of a length of $10 \mathrm{~mm}$.

\section{PULSED BESSEL BEAMS}

PBBs are coherent superpositions of monochromatic Bessel beams $A(\omega, z) J_{m}\left(k_{\perp} r\right) \exp (i m \phi) \exp (-i \omega t)$ of different frequencies $\omega$, where $(r, \phi)$ are polar coordinates in a plane perpendicular to the propagation direction $z, J_{m}()$ is the Bessel function of the $m$ th order, $A(\omega, z)$ $=A(\omega) \exp \left[i k_{z}(\omega) z\right]$, and

$$
k_{z}(\omega)=\left[k^{2}(\omega)-k_{\perp}^{2}\right]^{1 / 2},
$$

is the axial wavenumber. The transverse wavenumber $k_{1}>0$ is taken to be independent of frequency for PBBs

, which makes them factorized in time and space, with an identical Bessel profile at any temporal slice of the pulse, as we will see in Figs. 3 and 5.

Here we investigate the dispersion effects on the pulse propagation driven by the transversal localization of the $\mathrm{PBB}$, so that the refractive index $n$ is assumed to be independent of frequency for simplicity, or the propagation constant $k(\omega)=\omega n / c$ linear with frequency. From Eq. (1) the axial wavenumber $k_{z}$ vanishes at the cutoff frequency $\omega_{c}=k_{\perp} c / n$, taking real positive values $0<k_{z}<k$ for $\omega$ $>\omega_{c}$. The spectrum $A(\omega)$ is usually assumed to vanish for $\omega \leqslant \omega_{c}$.

For quasi-monochromatic radiation of carrier frequency $\omega_{0}$, the group velocity $v_{g}=1 / k_{z 0}^{\prime}$ along the $z$ direction (the prime stands for derivation with respect to $\omega$, and the subscript 0 for evaluation at $\omega_{0}$ ) results to be

$$
v_{g}=\frac{c}{n}\left[1-\left(\frac{\omega_{c}}{\omega_{0}}\right)^{2}\right]^{1 / 2},
$$

which is subluminal (lower than $c / n$ ). On the contrary, the phase velocity $v_{p}=\omega_{0} / k_{z 0}$ is found to be superluminal, and related to the group velocity by $v_{p} v_{g}=(c / n)^{2}$. Figure 1 shows the dispersion curve [Eq. (1)] in the plane $\omega-k_{z}$, where the relation $v_{g}<v_{p}$ is evidenced geometrically. Furthermore the slope of the dispersion curve approaching zero in the vicinity of $\omega_{c}$ evidences that PBBs can propagate at arbitrarily small group velocities. The speeds of the wave packet can be equivalently expressed in terms of

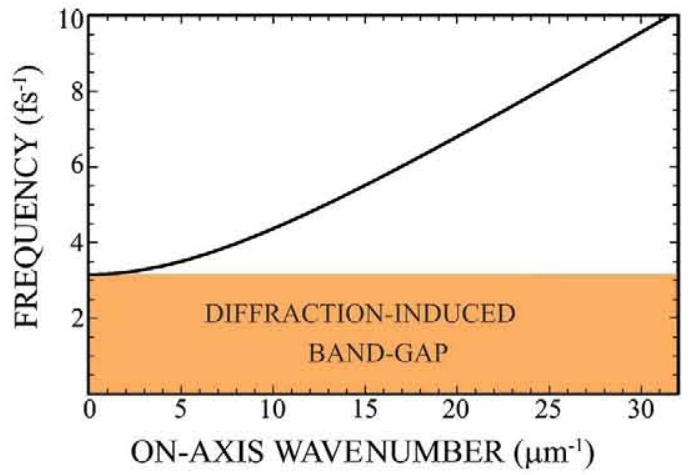

Fig. 1. (Color online) Typical $\omega-k_{z}$ diagram with a cutoff frequency of $\omega_{c}=3.14 \mathrm{fs}^{-1}$ and $n=1$. Bandgap induced by angular dispersion is filled in color. The slope of the curve at any point $\left(\omega, k_{z}\right)$ of the curve (or group velocity) is smaller than the quotient between $\omega$ and $k_{z}$ (phase velocity). the effective refractive index $n_{\text {eff }}=c / v_{p}$ and of the group index $n_{g}=c / v_{g}$, related by $n_{\text {eff }} n_{g}=n^{2}$ for PBBs. The group index is inversely proportional to the group velocity, and directly proportional to the phase velocity for PBBs. Value $n_{g} \gg n$ refers to the ultraslow propagation regime.

\section{ULTRAHIGH GROUP-INDEX REGIME}

In the vicinity of the band edge $\left(\omega \rightarrow \omega_{c}, k_{z} \rightarrow 0\right)$, the group velocity tends to zero, and hence the group index tends to infinity. $\mathrm{PBBs}$ in this ultraslow regime approach a nearly frozen pulse with a diffraction-free Bessel transversal profile. The dispersion relation in this regime can be approached by the parabola

$$
\omega=\omega_{c}\left(1+\frac{1}{2} \frac{k_{z}^{2}}{k_{\perp}^{2}}\right)=\omega_{c}+\frac{c^{2}}{2 \omega_{c} n^{2}} k_{z}^{2},
$$

which holds for a sufficiently low axial wavenumber $k_{z}$ $\ll k_{\perp}=\omega_{c} n / c$, or sufficiently small frequency shift $\omega-\omega_{c}$ from the cut of frequency. It follows from Eq. (3) that in this regime $v_{g}$ is proportional to the axial wavenumber $k_{z 0}$, and thus is proportional to the squared root of the shift $\delta \omega=\omega_{0}-\omega_{c}$ of the carrier frequency from the cutoff frequency $\omega_{c}$.

The proximity of slow-PBBs spectra to the band edge strongly influences the practicable pulse durations. If the spectrum is located about $\omega_{0}$ and must vanish for $\omega<\omega_{c}$, then the half-bandwidth $\sigma$ must satisfy $\sigma<\delta \omega$. From Eq. (3), $\delta \omega \approx\left(n^{2} / 2 n_{g}^{2}\right) \omega_{0}$, from which the fractional bandwidth $\Gamma \equiv 2 \sigma / \omega_{0}$ must satisfy $\Gamma<n^{2} / n_{g}^{2}$. Since $n_{g} \gg n$ in the ultraslow regime, ultraslow $\mathrm{PBB}$ are inherently quasimonochromatic $(\Gamma \ll 1)$. The number of cycles is roughly estimated by $N=\Gamma^{-1}$ (for a transform-limited pulse), which increases as the square root of the group index.

Although ultraslow PBBs are narrowband, the waveform may be distorted noteworthily upon propagation due to the strong dispersive character of the group index near the band edge. Figure 2 shows fast growth of group index and group-index dispersion [c times group-velocity dispersion (GVD)] collectively as $\omega_{0}$ approaches $\omega_{c}$. After traveling a distance $L$, the wave packet arrives with a time delay $T=L / v_{g}=(L / c) n_{g}$ varying for different spectral components of the field, and therefore causing pulse deformation if $L$ is large enough. Of particular relevance is the dispersion length $L_{D}$, or propagation distance at which the pulse becomes significantly distorted due to group-index dispersion. A significant distortion is ex-

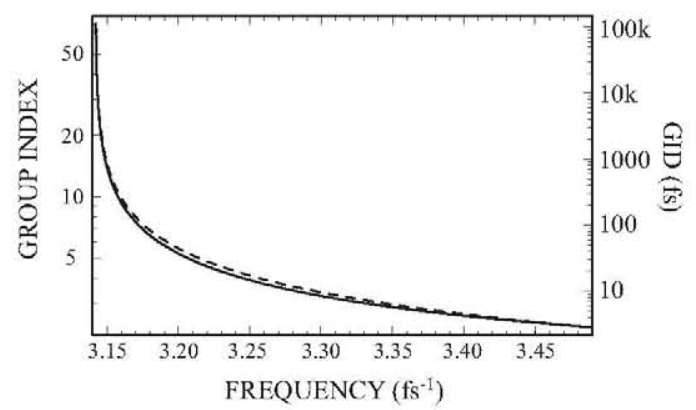

Fig. 2. Group index $n_{g}$ (solid curve) and group-index dispersion $n_{g}^{\prime}=k_{z 0}^{\prime \prime} c$ (dashed curve) for $\omega_{c}=3.14 \mathrm{fs}^{-1}$ in vacuum. 
pected to occur when the intraband delay $\Delta T=T\left(\omega_{0}+\sigma\right)$ $-T\left(\omega_{0}\right)$ is similar to the pulse duration $\tau=2 / \sigma$, which yields the usual expression $L_{D}=\tau^{2} / 2\left|k_{z 0}^{\prime \prime}\right|$ for the dispersion length. For ultraslow PBBs, Eq. (3) implies that $k_{z} k_{z}^{\prime}=\omega_{c} n^{2} / c^{2}$ and $k_{z} k_{z}^{\prime \prime}=-k_{z}^{\prime 2}$, which gives

$$
k_{z 0}^{\prime \prime}=-\frac{\omega_{c}^{2} n_{g}^{3}}{\omega_{0}^{3} n^{2} c} \approx-\frac{n_{g}^{3}}{\omega_{0} n^{2} c},
$$

and a dispersion length

$$
L_{D}=\frac{4 \lambda_{0} n^{2}}{\pi \Gamma^{2} n_{g}^{3}},
$$

where $\lambda_{0}=2 \pi c / \omega_{0}$ is the vacuum carrier wavelength. For the shortest PBB with given group index $n_{g}\left(\Gamma=n^{2} / n_{g}^{2}\right)$, the dispersion length becomes $L_{D}=4 \pi^{-1} \lambda_{0} n_{g} n^{-2}$, which is proportional to the group index and exceeds the carrier wavelength in several orders of magnitude.

In Sections 4 and 5 we take advantage of these basic properties of ultraslow PBBs for the design of light delay and storage devices without the need for any dispersive material medium or waveguiding structure. A commitment between group-velocity reduction and pulse duration may lead a simple dielectric slab (e.g., vacuum) to act as a delay line for femtosecond pulses with moderately slow velocities, or as a resonator with extremely slow pulses of nanosecond duration.

\section{FEW-CYCLE PULSE DELAY LINES}

We consider a dielectric slab of width $L$, with the left face at $z=0$ and an index of refraction of $n_{2}$, bounded by a dielectric cladding of refractive index $n_{1}>n_{2}$. The PBB of monochromatic components

$$
\psi_{m 1}^{+}=A_{1}^{+}(\omega, 0) J_{m}\left(k_{\perp} r\right) \exp (i m \phi),
$$

carrier frequency $\omega_{0}$, and transverse wavenumber $k_{\perp}$ impinges normally on the left face of the slab. The time origin is taken when the envelope of the input PBB is maximum at $z=0$. The Snell law of refraction implies that the Bessel transverse wavenumber is preserved within the dielectric slab.

The pulse bandwidth is limited by the cutoff frequency $\omega_{c}=k_{\perp} c / n_{2}$ of the slab, since it is is higher than the cutoff frequency of the cladding. The axial wavenumbers $k_{z j}(\omega)$ $=\left(n_{j}^{2} \omega^{2} / c^{2}-k_{\perp}^{2}\right)^{1 / 2}(j=1$ in the cladding at $z<0, j=2$ in the slab, and $j=3$ at $z>L$, with $n_{3}=n_{1}$ ) will be real and positive if the bandwidth of the input PBB satisfies $\sigma<\delta \omega$ $=\omega_{0}-\omega_{c}$. Within the $j$ domain, solutions of the wave equation are expressed as a superposition of the forwardpropagating wave of spectrum

$$
A_{j}^{+}(\omega, z)=A_{j}^{+}(\omega, 0) \exp \left(i k_{z j} z\right)
$$

and the backpropagating wave of spectrum

$$
A_{j}^{-}(\omega, z)=A_{j}^{-}(\omega, 0) \exp \left(-i k_{z j} z\right) .
$$

For simplicity in the boundary conditions, we consider PBBs whose axial electric field vanishes. In this case, the boundary conditions require continuity of the wave field and its normal derivative at interfaces $z=0$ and $L$. If a single wave packet impinges on the dielectric slab from the left side (i.e., $A_{3}^{-}=0$ ), the electric field may be expressed at any observation distance in terms of the spectrum $A_{1}^{+}(\omega, 0)$ of the incident field at $z=0$. In particular, the transmitted field at the output plane $z=L$ is seen to be given by

$$
\psi_{m 3}=\frac{A_{1}^{+}(\omega, 0) T_{1} T_{2} \exp \left(i k_{z 2} L\right)}{1-R^{2} \exp \left(i 2 k_{z 2} L\right)} J_{m}\left(k_{\perp} r\right) \exp (i m \phi),
$$

where

$$
\begin{gathered}
T_{1}=\frac{2 k_{z 1}}{k_{z 2}+k_{z 1}}, \\
T_{2}=\frac{2 k_{z 2}}{k_{z 2}+k_{z 1}}, \\
R=\frac{k_{z 2}-k_{z 1}}{k_{z 2}+k_{z 1}} .
\end{gathered}
$$

The set of equations (10) corresponds to the well-known Fresnel formulas for $s$-polarized states, where the difference $k_{z 2}-k_{z 1}$ is referred to as the impedance mismatch at the slab-cladding interfaces.

A convenient interpretation of Eq. (9) is commonly given by expanding $\psi_{m 3}$ into a power series of $R$ by use of the expression

$$
\left[1-R^{2} \exp \left(i 2 k_{z 2} L\right)\right]^{-1}=1+\sum_{q=1}^{\infty} R^{2 q} \exp \left(i 2 q k_{z 2} L\right) .
$$

This expansion suggests that the transmitted wave is a pulse train consisting of (1) a precursor field having the same waveform as the incident $\mathrm{PBB} \psi_{1}^{+}(\omega, 0)$, phase delay $k_{z 2} L$ and attenuation $T_{1} T_{2}$, and (2) an infinite number of wavelets originated from $2 q$ reflections at the slab interfaces at a reflectance rate $R$. The precursor arrives at $z$ $=L$ at a time $T=L / v_{g 2}$, where $v_{g 2}=1 / k_{z 2}^{\prime}$ (evaluated at $\omega$ $\left.=\omega_{0}\right)$ is the group velocity of the PBB in the slab. If the impedance at the interfaces is quasi-matched $\left(k_{z 2} \leqslant k_{z 1}\right)$, which corresponds to close enough refractive indices of slab and cladding, $R \approx\left(k_{z 2}-k_{z 1}\right)\left(2 k_{z 2}\right)^{-1}$ is close to zero and $T_{1} T_{2}=1-R^{2}$ approaches the unity. In this case, the precursor carries most of the energy from the incident $\mathrm{PBB}$, leading to a single strong signal at the output plane.

We perform numerical simulations in order to verify the validity of this analysis. From a cladding of refraction index $n_{1}=1.5$, we launched on the slab of refraction index $n_{2}=1.48$ the azimuthal $\mathrm{PBB}$ of spectrum

$$
\mathbf{E}=E_{\phi} \mathbf{u}_{\phi}=A_{1}^{+}(\omega, z) J_{1}\left(k_{\perp} r\right) \mathbf{u}_{\phi},
$$

about the typical optical frequency $\omega_{0}=3.14 \mathrm{fs}^{-1}$ and of duration $\tau=200 \mathrm{fs}$. The electric field in Eq. (12) is a diffraction-free solution of the reduced wave equation $\nabla$ $\times \nabla \times \mathbf{E}-k^{2} \mathbf{E}=0$ with zero axial components We point out that the azimuthal unitary vector $\mathbf{u}_{\phi}=-\sin \phi \mathbf{x}$ $+\cos \phi \mathbf{y}$ carries the angular dependence of the field over $\phi$. Since $\Gamma=6.37 \times 10^{-3}<n^{2} / n_{g}^{2}$ for PBBs, this femtosecond optical pulse can be transmitted as a PBB with a maximum group index $n_{g} \approx 18$. For the choice of the (rather 
strong) lateral localization $k_{\perp}=15.2 \mu \mathrm{m}^{-1}$ $\left(\gg k_{z 2}=2.87 \mu \mathrm{m}^{-1}\right.$ ), the cutoff frequency results to be $\omega_{c}$ $=3.083 \mathrm{fs}^{-1}$, and the group index takes the moderate value $n_{g}=8$. Finally, the thickness of the slab is chosen to be $L=20 \mu \mathrm{m}$, significantly smaller than the dispersion length $L_{D}=80.64 \mu \mathrm{m}$ in order to prevent any significant distortion of the transmitted PBB.

In Fig. 3(a) we represent the waveform of the field envelope $\left|E_{\phi}\right|^{2}$ at the input plane $z=0$. For convenience we used the Gaussian spectrum

$$
A_{1}^{+}\left(\omega_{0}+\Omega, 0\right)=\left(2 \pi \sigma^{2}\right)^{-1 / 2} \exp \left(-\Omega^{2} / 2 \sigma^{2}\right),
$$

since it leads to the analytical expression

$$
E_{\phi}(t, r)=\exp \left(-i \omega_{0} t\right) \exp \left(-t^{2} \sigma^{2} / 2\right) J_{1}\left(k_{\perp} r\right)
$$

for the incident wave field (if the spectral amplitude at $\omega_{c}$ is negligible). In Fig. 3(b) we show the transmitted field at $z=L$. The precursor arrives with a group delay of $T$ $=533 \mathrm{fs}$, a time $124 \mathrm{fs}$ longer than the time taken by the PBB in traveling the same distance $L$ within the homogeneous dielectric medium of refractive index $n_{1}$. Due to the quasi-matched impedances, the retarded wavelets originated from reflections at the interfaces cannot be observed at the scale of the figure, as expected from the energy balance given by $R=0.145$ (evaluated at $\omega=\omega_{0}$ ).

\section{ULTRASLOW RESONANCES}

We consider now the feasibility of PBBs with $n_{g}$ exceeding the unity by several orders of magnitude. For sufficiently small refractive index $n_{2}$ of the dielectric slab and same cladding, the impedance mismatch at the input and output interfaces is high, and the reflectivity $R$ may reach absolute values around the unity [see Eq. (10c)]. This attractive case leads to a leaky trapping of a extremely slow PBB within the layer through multiple reflections at $z$ $=0$ and $L$. Here we better speak of a resonator of the Fabry-Pérot type sustaining ultraslow PBBs rather than of a delay line.
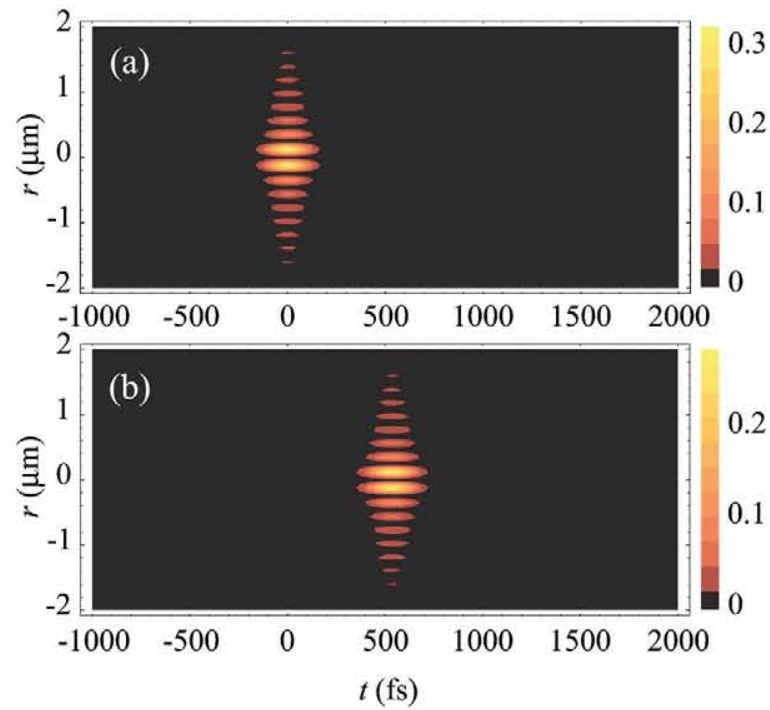

Fig. 3. (Color online) Instantaneous intensity $\left|E_{\phi}\right|^{2}$ (in arbitrary units) of the azimuthal PBB: (a) input plane $(z=0)$, and (b) output pulse $(z=L)$.
The round-trip group delay, or time consumed by the $\mathrm{PBB}$ in coming back to the input plane $z=0$ after being reflected at $z=L$, is given by $2 T=2 L / v_{g 2}$. For simplicity, we address our discussion to the central plane $z=L / 2$, where the released $\mathrm{PBB}$ arrives with a delay $T / 2$ and returns from a reflection on an interface with a periodic delay $T$. Replicas of the input beam propagating alternatively in the forward and backward directions are observed at a rate $T^{-1}$ in the form of a genuine pulse train. However, if the pulse duration $\tau$ is sufficiently long such that $\tau>T$, the leading part of the pulse moving backwards from reflection reaches the plane $z=L / 2$ in time to interfere with the rear part of the forward pulse. Overlapping will be negligible only if the resonator length is larger than the pulse coherence length, that is,

$$
L>L_{c}=\tau_{g 2}=\frac{2 \lambda_{0}}{\pi n_{g} \Gamma} .
$$

Moreover nontotal reflection at the interfaces and dispersive distortion at each round trip causes attenuation and broadening of the replicas at distances greater than the dispersion length $L_{D}$ in Eq. (5). Since $L_{D}$ and $L_{c}$ are related by $L_{c}=L_{D} \Gamma n_{g}^{2} /\left(2 n_{2}^{2}\right)$, and the bandwidth is limited to $\Gamma<n_{2}^{2} / n_{g}^{2}$, the dispersion length is found to satisfy $L_{D}$ $>2 L_{c}$. As a conclusion, a range of resonator lengths $L$ $\in\left(L_{c}, L_{D}\right)$ exists in which overlapping and distortion are simultaneously negligible, at least for a number of round trips $\sim L_{D} /(2 L)$. This number can be significantly high as long as $L \geqslant L_{c}$ and $\Gamma \ll n_{2}^{2} n_{g}^{-2}$.

As a particular case, we consider again the azimuthal PBBs in Eq. (12) as the light signal that excites a resonant $\mathrm{PBB}$ when it is launched from the left side of the cladding $\left(n_{1}=1.5\right)$ onto a vacuum cavity $\left(n_{2}=1\right)$, a configuration that provides high reflectivity at the interfaces. For the visible carrier frequency $\omega_{0}=3.14 \mathrm{fs}^{-1}$, Fig. 4(a) shows the group index reachable as a function of its detuning $\delta \omega$ from the cutoff frequency. For a choice of the ultrahigh group index $n_{g}=10^{3}$, the detuning must be $\delta \omega=1.57$ $\times 10^{6} \mathrm{fs}^{-1}$, attained by a PBB of transverse frequency $k_{\perp}$ $=10.5 \mu \mathrm{m}^{-1}$, or a transversal spot size of about $0.2 \mu \mathrm{m}$. In Fig. 4(b) we plot the dispersion length $L_{D}$ and the coherence length $L_{c}$ for the different possible bandwidths $\sigma$ $<\delta \omega$ of the PBB, where $L_{c}<L_{D}$ is evidenced graphically. For instance, a pulse duration of $\tau=20 \mathrm{~ns}\left(\sigma=10^{-7} \mathrm{fs}^{-1}\right.$, or $\Gamma=6.37 \times 10^{-8}$ ) yields $L_{c}=6 \mathrm{~mm}$ and $L_{D}=18.85 \mathrm{~cm}$. A cav-
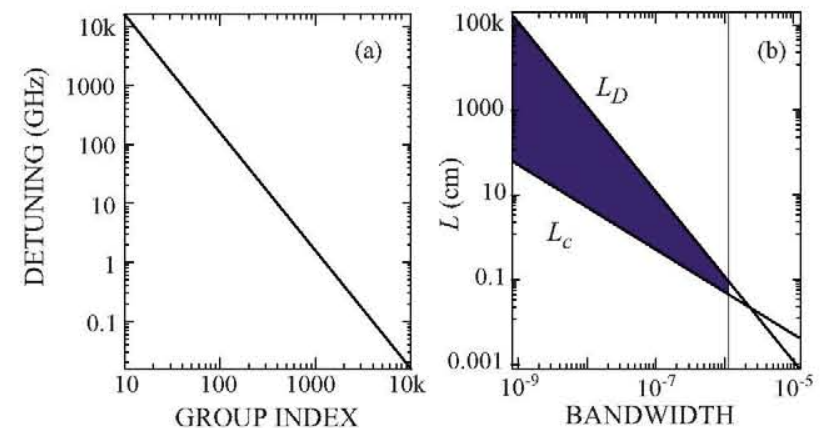

Fig. 4. (Color online) Diagrams for the synthesis of ultraslow resonant PBBs. (a) Spectral detuning $\delta \omega=\omega_{0}-\omega_{c}$ required to attain a given group index for $\omega_{0}=3.14 \mathrm{fs}^{-1}$. (b) Region $L_{c}<L<L_{D}$ (in color) as for allowable relative bandwidths $\Gamma$. 
ity of length $L=10 \mathrm{~mm}$, as considered below in numerical simulations, would result in nearly 10 shapely round trips without overlapping and distortion, with a period of $2 T=66.7 \mathrm{~ns}$. The ultraslowness of the PBB along with the pseudoresonance provides a storage time of the order of the microsecond, to be compared with the fly time of $\sim 0.3 \times 10^{-11} \mathrm{~s}$ of light in vacuum.

In Fig. 5 we show contour plots of the intensity $\left|E_{\phi}\right|^{2}$ at the input and midplane of the resonator. The intracavity field is computed following the procedure described in Section 4 based on the continuity conditions on the interfaces, which leads to the in-plane spectra

$$
\begin{aligned}
& A_{2}^{+}(\omega, 0)=\frac{T_{1}}{1-R^{2} \exp \left(i 2 k_{z 2} L\right)} A_{1}^{+}(\omega, 0), \\
& A_{2}^{-}(\omega, 0)=\frac{R T_{1} \exp \left(i 2 k_{z 2} L\right)}{1-R^{2} \exp \left(i 2 k_{z 2} L\right)} A_{1}^{+}(\omega, 0),
\end{aligned}
$$

for the forward-propagating and counterpropagating components of the $\mathrm{PBB}$, respectively, where $A_{1}^{+}(\omega, 0)$ is given by the Gaussian signal in Eq. (13). Figure 5(a) shows a field at the input face $z=0$ of the resonator. Only the input excitation is observable at the scale of the figure since multiple reflections are strongly attenuated. This effect originates from the negative sign of the reflection coefficient $R=-0.998$, which causes the leading part of any reflected pulse to interfere destructively with the rear part of the pulse prior to reflection. The strongly attenuated reflections are more clearly seen in Fig. 5(c) for the output face $z=L$. Attenuation is seen to be significant up
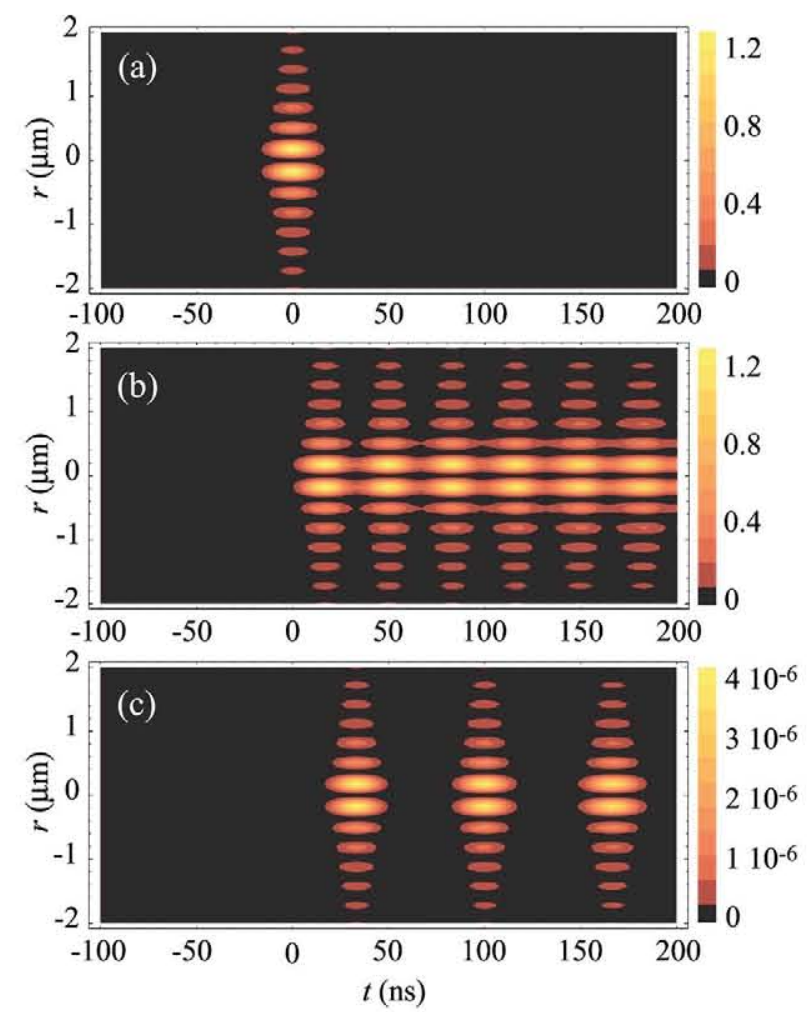

Fig. 5. (Color online) Intracavity dynamics of $\left|E_{\phi}\right|^{2}$ at different transverse planes: (a) input plane $z=0$, (b) amid-reflectors plane $z=L / 2$, and (c) interface plane $z=L$. to distances $L_{c}$ from the faces of the resonator, which yields an effective cavity length of about $L-2 L_{c}$ without attenuation. Figure 5(b) shows that the field at the central plane $z=L / 2$ of the resonator is composed of a periodic sequence of nonoverlapping, nearly undistorted, and nonattenuated $\mathrm{PBBs}$ of period $T=33.3 \mathrm{~ns}$ and an offset group delay of $T / 2$. Time reversal of alternate pulses is not appreciated due to the symmetry of the Gaussian pulse.

\section{CONCLUSIONS}

In this paper we have established the conditions for the observation and synthesis of wave packets with ultraslow group velocity in a nondispersive medium or free space without the help of any guiding structure. In practice, laser emission from surface-emitting semiconductors carries the required angular dispersion to produce subluminal pulsed Bessel beams, which demonstrates that nearfield diffraction of nonevanescent waves allows practical realizations of ultraslow wave velocities in free space. Drastic reduction of the group velocity is achieved by strong angular dispersion of the composing monochromatic Bessel beams at angles close to $\pi / 2$, or, equivalently, by pseudostanding waves with transversal wavenumbers much larger than the axial wavenumber, leading additionally to strong transverse localization into a few nanometers.

The analysis of the limitations in PBB bandwidth and dispersive distortion, as imposed by the presence of the bandgap, allows us to conclude that a femtosecond optical PBB can propagate at moderate slow velocities $\left(n_{g} \sim 10\right)$, while extremely slow group velocities $\left(n_{g} \sim 10^{3}\right.$ or larger) are possible with picosecond or longer pulses.

We conclude our investigation with the analysis of nonguiding, uniform delay lines, based on lowering the refraction index with respect to the surrounding medium, which hence slow down the exciting pulsed Bessel beam. For convenience, azimuthally symmetric vector Bessel beams have been taken for the analysis and numerical simulations. The influence of the refractive index mismatch at input and output planes onto the pulse dynamics is examined. When the index mismatch is sufficiently low the dielectric slab emulates a lossless delay line usable for few-cycle femtosecond pulses. An increase of the mismatch enhances the reflectivity at the interfaces, so that the plate (vacuum in our numerical simulations) acts as an optical resonator sustaining an extremely slow PBB of nanosecond duration. Our analysis leaves an open door for the experimental demonstration and optical engineering of ultraslow wave phenomena in free space.

\section{ACKNOWLEDGMENTS}

This research was funded by the Ministerio de Educacion y Ciencia (grant HU2007-0020) and the Generalitat Valenciana (grants GV/2007/043 and GVPRE/2008/005). Carlos J. Zapata-Rodríguez also acknowledges financial support from the Universitat de València and Ministerio de Ciencia e Innovación (grant PR2007-0324). 


\section{REFERENCES}

R. W. Boyd and D. J. Gauthier, Slow and Fast Light, Progress in Optics, Vol. 43 of E. Wolf, ed. (Elsevier, 2002), chap. 6, pp. 497-530.

L. V. Hau, S. E. Harris, Z. Dutton, and C. H. Behroozi, "Light speed reduction to 17 metres per second in an ultracold atomic gas," Nature 397, 594-598 (1999).

M. D. Lukin and A. Imamoğlu, "Controlling photons using electromagnetically induced transparency," Nature 413, 273-276 (2001)

M. Artoni, G. C. La Rocca, F. S. Cataliotti, and F. Bassani, "Highly anomalous group velocity of light in ultracold rubidium gases," Phys. Rev. A 63, 023805 (2001).

M. S. Bigelow, N. N. Lepeshkin, and R. W. Boyd, "Superluminal and slow light propagation in a roomtemperature solid," Science 301, 200-202 (2003).

J. A. Stratton, Electromagnetic Theory (Mcgraw-Hill College, 1941).

M. Zamboni-Rached, K. Z. Nóbrega, E. Recami, and H. E. Hernández-Figueroa, "Superluminal X-shaped beams propagating without distortion along a coaxial guide," Phys. Rev. E 66, 046617 (2002).

M. Zamboni-Rached, F. Fontana, and E. Recami, "Superluminal localized solutions to Maxwell equations propagating along a waveguide: the finite-energy case," Phys. Rev. E 67, 036620 (2003).

L. Brillouin, Wave Propagation and Group Velocity (Academic, 1960).

T. M. Wallett and A. H. Qureshi, Review of Slow-Wave Structures, NASA Technical Memorandum 106639 (NASA, 1994), pp. 1-28.

S. Wang, H. Erlig, H. R. Fetterman, E. Yablonovitch, V. Grubsky, D. S. Starodubov, and J. Feinberg, "Measurement of the temporal delay of a light pulse through a onedimensional photonic crystal," Microwave Opt. Technol. Lett. 20, 17-21 (1999).

H. Altug and J. Vucković, "Experimental demonstration of the slow group velocity of light in two-dimensional coupled photonic crystal microcavity arrays," Appl. Phys. Lett. 86, 111102 (2005).

H. Gersen, T. J. Karle, R. J. P. Engelen, W. Bogaerts, J. P. Korterik, N. F. van Hulst, T. F. Krauss, and L. Kuipers, "Real-space observation of ultraslow light in photonic crystal waveguides," Phys. Rev. Lett. 94, 073903 (2005).

J. G. Rivas, A. F. Benet, J. Niehusmann, P. H. Bolivar, and H. Kurz, "Time-resolved broadband analysis of slow-light propagation and superluminal transmission of electromagnetic waves in three-dimensional photonic crystals," Phys. Rev. B 71, 155110 (2005).

M. L. Povinelli, S. G. Johnson, and J. D. Joannopoulos, "Slow-light, band-edge waveguides for tunable time delays," Opt. Express 13, 7145-7159 (2005).

L. H. Frandsen, A. V. Lavrinenko, J. Fage-Pedersen, and P. I. Borel, "Photonic crystal waveguides with semi-slow light and tailored dispersion properties," Opt. Express 14, 9444-9450 (2006).

A. Melloni, F. Morichetti, and M. Martinelli, "Optical slow wave structures," Opt. Photonics News 14, 44-48 (2003).

J. Durnin, J. J. Miceli, and J. H. Eberly, "Diffraction-free beams," Phys. Rev. Lett. 58, 1499-1501 (1987).

J. Lu and J. F. Greenleaf, "Nondiffracting X waves - exact solutions to free-space scalar wave equation and their finite aperture realizations," IEEE Trans. Ultrason. Ferroelectr. Freq. Control 39, 19-31 (1992).

P. Saari, "Localized waves in femtosecond optics" (2003), personal communication.

S. Licul, "On the generation and applications of localized waves," M.Sc. thesis (Virginia Polytechnic Institute, 2001). H. E. Hernández-Figueroa, M. Zamboni-Rached, and E. Recami, Localized Waves (Wiley, 2008).

M. A. Porras, G. Valiulis, and P. D. Trapani, "Unified description of Bessel $\mathrm{X}$ waves with cone dispersion and tilted pulses," Phys. Rev. E 68, 016613 (2003).

C. J. Zapata-Rodríguez and M. A. Porras, "X-wave bullets with negative group velocity in vacuum," Opt. Lett. 31, 3532-3534 (2006).

I. Alexeev, K. Kim, and H. M. Milchberg, "Measurement of the superluminal group velocity of an ultrashort Bessel beam pulse," Phys. Rev. Lett. 88, 073901 (2002).

E. Recami, M. Zamboni-Rached, K. Z. Nóbrega, C. A. Dartora, and H. E. Hernández, "On the localized superluminal solutions to the Maxwell equations," IEEE J. Sel. Top. Quantum Electron. 9, 59-73 (2003).

P. Saari and K. Reivelt, "Evidence of X-shaped propagationinvariant localized light waves," Phys. Rev. Lett. 79, 4135-4138 (1997).

H. Sönajalg, M. Rätsep, and P. Saari, "Demonstration of the Bessel-X pulse propagating with strong lateral and longitudinal localization in a dispersive medium," Opt. Lett. 22, 310-312 (1997)

M. A. Porras, "Diffraction-free and dispersion-free pulsed beam propagation in dispersive media," Opt. Lett. 26, 1364-1366 (2001).

Z. Liu and D. Fan, "Propagation of pulsed zeroth-order Bessel beams," J. Mod. Opt. 45, 17-22 (1998).

C. J. R. Sheppard, "Generalized Bessel pulse beams," J. Opt. Soc. Am. A 19, 2218-2222 (2002).

M. Zamboni-Rached and E. Recami, "Subluminal wave bullets: exact localized subluminal solutions to the wave equations," Phys. Rev. A 77, 033824 (2008).

M. Zamboni-Rached, "Stationary optical wave fields with arbitrary longitudinal shape by superposing equal frequency Bessel beams: frozen waves," Opt. Express 12, 4001-4006 (2004).

C. J. R. Sheppard, "Bessel pulse beams and focus wave modes," J. Opt. Soc. Am. A 18, 2594-2600 (2001).

B. Lü and Z. Liu, "Propagation properties of ultrashort pulsed Bessel beams in dispersive media," J. Opt. Soc. Am. A 20, 582-587 (2003).

A. M. Shaarawi, "Comparison of two localized wave fields generated from dynamic apertures," J. Opt. Soc. Am. A 14, 1804-1816 (1997).

C. J. Zapata-Rodríguez and M. T. Caballero, "Isotropic compensation of diffraction-driven angular dispersion," Opt. Lett. 32, 2472-2474 (2007).

C. J. Zapata-Rodríguez, M. T. Caballero, and J. J. Miret, "Angular spectrum of diffracted wavefields with apochromatic correction," Opt. Lett. 33 1753-1755 (2008). C. J. Zapata-Rodríguez, "Focal waveforms with tunable carrier frequency using dispersive aperturing," Opt. Commun. 281, 4840-4843 (2008).

L. Niggl, 'T. Lanzl, and M. Maier, "Properties of Bessel beams generated by periodic gratings of circular symmetry," J. Opt. Soc. Am. A 14, 27-33 (1997).

S. Holm, "Bessel and conical beams and approximation with annular arrays," IEEE Trans. Ultrason., Ferroelect., IEEE 'Trans. Ultrason. Ferroelectr. Freq. Control 45, 712-718 (1998).

T. Erdogan, O. King, G. W. Wicks, D. G. Hall, E. H. Anderson, and M. J. Rooks, "Circularly symmetric operation of a concentric-circle-grating, surface-emitting, AlGaAs/GaAs quantum-well semiconductor laser," Appl. Phys. Lett. 60, 1921-1923 (1992).

R. H. Jordan and D. G. Hall, "Highly directional surface emission from concentric-circle gratings on planar optical waveguides: the field expansion method," J. Opt. Soc. Am. A 12, 84-94 (1995).

P. Polesana, A. Couairon, D. Faccio, A. Parola, M. A. Porras, A. Dubietis, A. Piskarskas, and P. Di Trapani, "Observation of conical waves in focusing, dispersive, and dissipative Kerr media," Phys. Rev. Lett. 22, 223902 (2007). P. L. Greene and D. G. Hall, "Properties and diffraction of vector Bessel-Gauss beams," J. Opt. Soc. Am. A 15, 3020-3027 (1998). 